\title{
土壤生态系统中抗生素抗性基因与星球健康: 进展与展望
}

\author{
朱冬 ${ }^{1}$, 陈青林 $^{2}$, 丁晶 ${ }^{1}$, 王一飞 ${ }^{1}$, 崔慧灵 $^{1}$, 朱永官 $^{1,2 *}$ \\ 1. 中国科学院生态环境研究中心, 城市与区域生态国家重点实验室, 北京 100085 ; \\ 2. 中国科学院城市环境研究所, 中国科学院城市环境与健康重点实验室, 厦门 361021 \\ * 联系人, E-mail: ygzhu@rcees.ac.cn
}

收稿日期：2019-11-13; 接受日期：2019-12-02; 网络版发表日期：2019-12-10

国家自然科学基金(批准号: 41571130063)和国家重点研发计划政府间重点专项(批准号: 2017YFE0107300)资助

\begin{abstract}
摘要 土壤-植物系统是抗生素抗性基因(简称抗性基因)从环境向人类传播扩散的一个重要途径, 是环境抗性基 因人群暴露的主要来源. 通过对土壤-植物系统中抗性基因的研究，可以明确抗性基因在土壤生物中的分布及其 传递，分析抗性基因食物链传递的风险及其规律，从而为控制抗性基因污染提供理论依据，保障人类健康. 同时， 其对于维护星球健康也具有重要的价值. 本文总结了土壤(包括土壤动物肠道)和植物微生物组中抗性组的重要研 究进展, 强调了抗性基因流在土壤-植物系统中的流动给土壤生态系统带来的环境风险, 最后提出了通过管理土 壤-植物系统中的抗性基因流以保障星球健康的方案, 并对未来研究进行了展望.
\end{abstract}

关键词 土壤-植物系统, 星球健康, 抗生素抗性基因, 食物链传递, 人类活动

抗生素为人类防治细菌感染、保障动物畜牧养殖 以及农业生产做出了重大贡献. 然而, 随着抗生素的长 期大量使用和滥用, 抗生素耐药性问题日趋加剧, 已经 成为全球最严峻的公共卫生问题之一，严重威胁人类 健康. 世界卫生组织(World Health Organization, WHO)于2011年发出“遏制耐药一今天不采取行动, 明天就无药可用”的呼吁，并于2014年发布了“控制细 菌耐药全球行动计划(草案)”. 我国也于2016年由国家 卫生和计划生育委员会(2018年整合至国家卫生健康 委员会)等14部门联合制定并发布了《遏细菌耐药国 家行动计划(2016-2020年)》, 旨在加强抗菌药物科学 管理，遏制细菌耐药发展与蔓延，维护人民群众身体
健康, 促进经济社会协调发展.

环境中的抗生素残留即使在低浓度下也可以对细 菌产生选择压力. 随着人类活动(有机肥施用和污水灌 溉等)的加剧, 抗生素抗性细菌(以下简称抗性细菌)和 抗生素抗性基因(antibiotic resistance genes, ARGs, 以 下简称抗性基因)在环境中不断扩散增殖. 抗性基因已 被广泛认为是一类新型的环境污染物 ${ }^{[1]}$, 与传统化学 污染物不同，抗性基因污染属于生物污染，不仅会在 不同环境介质中持久性残留、转移和扩散, 而且具有 暴发性的特征, 一旦失控, 将严重威胁公共安全. 例如, 2010年印度新德里报道的“超级细菌”事件，是由于抗 生素对携带有抗性基因的致病菌治疗无效引发的 ${ }^{[2]}$.

引用格式: 朱冬, 陈青林, 丁晶, 等. 土壤生态系统中抗生素抗性基因与星球健康: 进展与展望. 中国科学: 生命科学, 2019, 49: 1652-1663 Zhu D, Chen Q L, Ding J, et al. Antibiotic resistance genes in the soil ecosystem and planetary health: Progress and prospect (in Chinese). Sci Sin Vitae, 2019, 49: 1652-1663, doi: 10.1360/SSV-2019-0267 
我国是抗生素的生产与消费大国，其引起的环境问题 一直受到中国科学家的广泛关注. 国内已对环境中的 抗生素与抗性基因污染的生态风险展开了系统的研 究, 并取得了很多突破性进展.

土壤-植物系统包括绿色植物及其根系周围的土 壤环境(土壤、微生物和动物), 是地球生态系统中与 人类生存和健康最为密切的系统, 其实质是涉及土壤微生物-动物-植物的四元体系. 土壤是地球上最大的 微生物栖息地, 同时也是最大的抗性基因储存库. 随 着人类活动影响的加剧, 粪肥、污泥农用及再生水灌 溉等因素使得土壤抗性基因水平显著升高 ${ }^{[3]}$. 生长在 土壤中的植物, 其表面和内部附着各种微生物, 统称 为植物微生物组, 它们在植物生长和抗病中发挥重要 作用. 植物的根际微生物和叶际微生物是植物微生物 组的重要组成. 植物根际环境是受植物根系和根系分 泌物直接影响的薄层土壤, 包含复杂的微生物类群, 根际微生物主要来源于土壤环境. 植物叶际微生物主 要来源于植物本身的土著微生物和通过土壤、空气及 其他植物水平传播获得的外源微生物 ${ }^{[4]}$. 土壤、根际 和叶际的微生物活动将土壤、微生物和植物联系起 来, 构成环境中一个独特的生态系统单元. 土壤-植物 系统是抗性基因从环境向人类传播扩散的重要途径之 一, 是环境抗性基因人群暴露的主要来源. 食物链是土 壤-植物系统中的抗性细菌和抗性基因进入人体最直 接和最主要的途径. 当人们摄入生食的蔬菜、水果等 时, 蔬果携带的抗性细菌和抗性基因将直接进入人体, 从而对人类健康造成潜在威胁 ${ }^{[5]}$. 随着全球经济的快 速发展, 农产品中的抗性基因随着食物的加工、保存 和全球运输, 可以迅速在全球范围内传播和扩散 ${ }^{[6]}$, 引 发公共安全风险, 从而影响星球健康.

面向人类未来可持续发展问题，2015《柳叶刀》

(The Lancet)杂志社组织全球专家形成了关于“星球健 康”的专题报告. 该报告指出, 星球健康是一个新的研 究领域, 它将自然环境与人类健康相联系 ${ }^{[7]}$. “星球健 康”认为, 人类健康及其福祉与星球系统有着千丝万缕 的联系, 需要保护自然环境的完整性, 以确保人类长期 持续的健康 ${ }^{[8]}$. “星球健康”特别强调将过程与现象相 联系, 可能这些现象与过程按照传统角度来看似乎没 有联系. 例如, 大气中 $\mathrm{CO}_{2}$ 浓度升高可能导致农作物微 量元素的稀释, 但人体摄入的农作物总量不变, 这可能 导致人体微量元素的缺乏 ${ }^{[9]}$. 因此, 需要多学科的交叉
研究来揭示复杂和跨领域的问题，从而更好地管理自 然生态系统, 保障人类的健康与福祉 ${ }^{[10]}$. 因此, 本文首 先总结了我国在土壤(包括土壤动物肠道)和植物微生 物组中抗性组的重要研究进展, 强调了抗性基因流在 土壤-植物系统中的流动, 最后提出了通过管理土壤植物系统中的抗性基因流来保障星球健康的方案, 并 提出了未来的研究方向.

\section{1 土壤微生物组中的抗生素抗性组}

\section{1 土壤是抗生素的重要来源}

土壤是地球上生物多样性最高的栖息地, 其中最 活跃的成分是土壤微生物, $1 \mathrm{~g}$ 土壤中包含上百万细 菌、数十万真菌孢子及数万原生动物. 这些微生物在 代谢过程中, 不断产生各种次级代谢产物, 用于种间 交流和资源竞争，如抗生素、毒素、色素等 ${ }^{[11]}$. 当前 临床中使用的大部分抗生素都来源于土壤中的细菌和 真菌, 其中放线菌是土壤中最大的细菌门之一, 占土壤 微生物种群的 $13 \% \sim 30 \%$, 产生了已知抗生素的 $2 / 3^{[12]}$. 根据结构特点可以将抗生素分为内酰胺、氨基糖苷、 核苷、四环素、多肽、糖肽、大环内酯等种类. 抗生 素可以抑菌杀菌外, 还可以作为抗癌药、抗寄生虫 药、除草剂等, 在医疗、工业、农牧渔业和环境保护 等领域均发挥重要作用. 但因抗生素大量滥用造成抗 性菌的蔓延和传统分离技术应用的限制, 抗生素的发 展进入了停滞期. 近年来, 随着分子生物学及多组学 技术的不断发展, 有望在土壤及其他环境中分离新的 活性物质, 突破抗生素发展的瓶颈 ${ }^{[13]}$.

\section{2 土壤是环境中抗生素抗性基因的重要储存库}

（1）抗性基因的本底值(内在抗性). 抗性菌对抗生 素的抗性来源于位于染色体或者可移动基因元件上的 抗性基因. 与传统的化学污染物不同, ARGs可在同种 甚至不同种细菌之间进行转移和传播, 影响抗生素的 治疗效果, 已被列为一种新型环境污染物 ${ }^{[14]}$. 抗性机 制主要包括以下4类(图1) ${ }^{[15]}$ : ( i ) 使抗生素失活: 降解 抗生素或取代活性基团; ( ii) 抗性突变: 突变修饰抗生 素的结合靶位(目标蛋白), 使抗生素结合位点失效, 但 保持蛋白质的细胞功能不变; (iii) 外排洜: 通过药物外 排洜将抗生素排出细胞; (iv) 多糖屏障: 在细胞膜上形 成屏障等. 


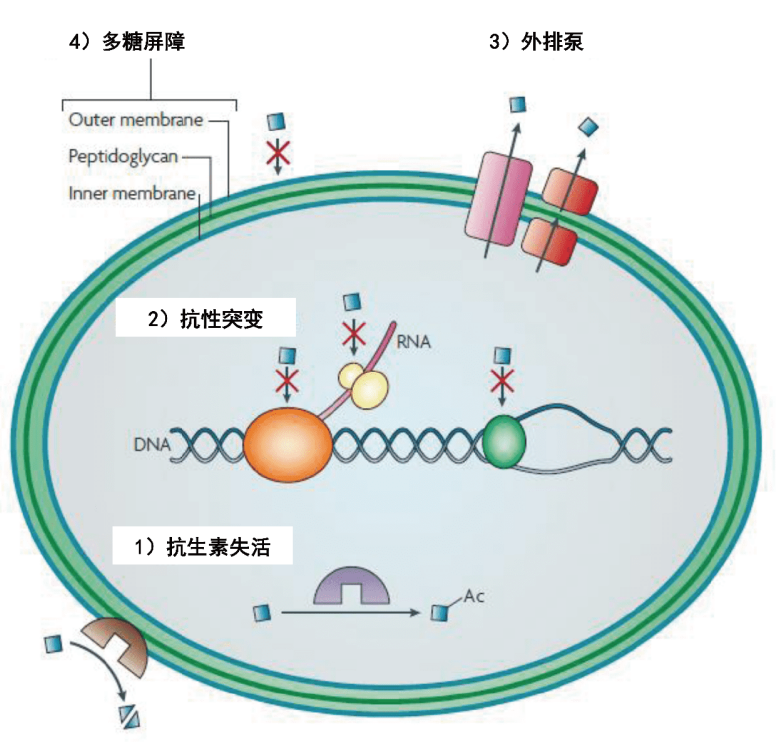

图 1 革兰氏阴性菌的抗生素耐性机制 ${ }^{[15]}$

Figure 1 Mechanisms of antibiotic resistance in a gram-negative bacterium $^{[15]}$

土壤环境中存在大量天然抗生素, 例如, 最早的抗 生素之一链霉素就来自于土壤. 尽管这些抗生素的浓 度很低, 但仍可作为微生物种群之间或种群内的信号 分子, 形成一定的环境压力, 使微生物种群获得抗性, 因此土壤中的微生物群落普遍存在内在抗性基因. 例 如, 在没有人为活动施加影响的阿拉斯加冻土中, Allen等人 ${ }^{[16]}$ 通过功能性宏基因组分析首次发现了双功 能的 $\beta$ 内酰胺酶基因. 这表明一些ARGs是自然存在于 土壤环境中的，与人类活动造成的选择压力无关. 对 30000年前冻土的DNA进行宏基因组分析, Galán等 人 $^{[17]}$ 发现, 内在抗性基因组具有高度多样性, 其中一 些基因编码对多种药物具有抗性，比如 $\beta$-内酰胺类、 糖肽和四环素类抗生素.

(2) 外源输入. 作为 20 世纪最重要的医学发现之 一, 抗生素自发现之日起就被大量投入生产和使用, 广 泛应用于人类医疗、动物畜牧养殖以及农业生产中. 目前, 在全球范围内, 抗生素滥用的情况十分严重. 根 据美国疾病预防控制中心统计，人类使用的抗生素中 有多达 $50 \%$ 是不必要或不恰当的. 抗生素滥用对细菌 造成了持续性的选择压力, 促进了ARGs在多种病原 体和共生微生物中的形成和扩散. 有研究表明, ARGs 在微生物之间主要通过由噬菌体介导的转导、可移动 性基因元件(mobile genetic elements)介导的接合及游
离DNA的转化进行传播. Peterson和Kaur ${ }^{[18]}$ 发现, 土壤 中的本土耐药微生物不再能代表土壤微生物种群中的 大多数耐药生物. 相反, 土壤中的微生物越来越多地从 人类活动中获得抗性.

污水处理厂是土壤中ARGs的主要人为来源. 医疗 废水、生活废水及工业废水中的抗生素、ARGs很难 在城市污水处理厂中被完全消除. 我国科学家在我国 污水处理厂的污泥及出水中检测到超过 200 类ARGs, 这些ARGs即会随着再生水灌溉、污泥施用进入城市 及农田土壤中 ${ }^{[19]}$. 例如，王凤花等人 ${ }^{[20]}$ 采用高通量定 量PCR技术在经再生水灌溉的公园土壤中检测出 147 种抗性基因, 这些基因与对广谱抗生素的抗性有关, 其 中最丰富的是抗 $\beta$ 抗内酰胺 $(18.4 \%$ 30\%)类、FCA类 (氟喹诺酮、喹诺酮、氟苯尼考、氯霉素、酰胺醇) (12.5\% 22\%)、氨基糖苷类(7.5\% 19.4\%)抗性基因, 与 未经再生水灌溉的对照土壤相比, 抗性基因丰度的富 集范围从99到8655倍不等, 值得注意的是, 多重耐药 类抗性基因如qacEdeltal-02 富集了800倍，这类抗性基 因在土壤环境中的扩散传播可能会引起微生物对其他 乃至新型抗生素的抗性, 导致抗生素失效; 施用污泥的 农田土壤中同样检测到较高丰度的抗性基因, 更为重 要的是, 一些抗性基因如 $\operatorname{aad} \mathrm{A}, \operatorname{tet}(\mathrm{W})$ 等在施用污泥 119 天后仍然可以检测到，表现出较高的传播风险，同 时高丰度的转座子intI 1也通过定量PCR检测到, 这类 整合子通常携带多个抗性基因, 可能会使抗性基因转 移到人类致病菌中, 危害人类健康 ${ }^{[21]}$.

抗生素在动物畜牧养殖业中大量应用, 有研究发 现，40\% 90\%的饲用抗生素以原化合物或具有生物活 性的代谢产物的形式随动物粪便排出; 另外, 抗生素的 应用会使动物肠道内积累丰富的ARGs ${ }^{[22]}$, 因此粪肥 农用可能是ARGs进入土壤环境的重要途径. 研究表 明, 在施用猪粪的农田土壤中, 检测到较高水平的 ARGs和MGEs(mobile genetic elements) ${ }^{[23]}$. 通过使用 高通量苂光定量PCR技术, 朱永官团队 ${ }^{[24]}$ 分析了国内 北京、浙江和福建三个大型商业养猪场附近土壤的抗 性基因, 发现因施粪肥引起的抗生素残留的土壤样品 中有 63 种ARGs的丰度显著高于对照土壤, 最高达 21600 倍; 同时MGEs的丰度与ARGs的总丰度呈显著 正相关, 这暗示着由MGEs介导的水平基因转移可能 是引起ARGs富集的重要原因.

空气污染也可能通过干湿沉降将ARGs引入土壤. 
我国学者调查研究了ARGs在全球 19 个城市空气中的 分布情况, 发现大环内酯类和喹诺酮类抗性基因是空 气中最丰富的ARGs，并强调了城市空气传播ARGs的 潜在风险 ${ }^{[25]}$. 最近的一项研究也发现, 北京、天津及 石家庄的城市灰尘样品中ARGs的浓度高达每克灰尘 106.4 拷贝，且会通过风力等因素进行传播 ${ }^{[26]}$. 这些在 空气中的ARGs和其他污染物可能会跨越地理障碍进 行长距离运输, 甚至沉降到偏远地区的土壤中, 从而加 剧土壤中ARGs的扩散. 同时, 土壤污染物和微生物也 可能被雾化到空气中, 并进一步加剧ARGs在空气中的 传播. ARGs通过颗粒物从土壤迁移到空气，高污染土 壤中ARGs在土-气中的迁移趋势更加明显，土壤含水 率升高会降低ARGs的土-气迁移趋势 ${ }^{[27]}$.

此外，土壤微生物对环境变化的响应存在物种差 异，这导致人类活动对ARGs的影响在不同地区也有 所区别. 如施用污泥堆肥后，土壤类型对抗性基因的 扩散有显著影响 ${ }^{[28]}$, 污泥堆肥 172 天后, 与对照组样品 相比, 江西红土中抗性基因的风险并未增加, 而山西黄 土、吉林黑土中的抗性基因则显著增加. 驱动不同土 壤抗性基因变化的因素也不尽相同，堆肥导致微生物 群落的变化是影响江西红土和吉林黑土中抗性基因变 化的主要因素，而山西黄土中的可移动基因元件占主 导地位，这表明堆肥处理在山西黄土中具有更高的抗 性传播风险. 同时, 除抗生素外,一些非抗生素类物质, 如重金属、人类药物等，会显著促进抗性基因在土壤 中的扩散 ${ }^{[29 \sim 31]}$. 胡行伟等人 ${ }^{[29]}$ 通过高通量定量PCR发 现，铜污染 $(0 \sim 800 \mathrm{mg} / \mathrm{kg}) 4 \sim 5$ 年的农田土壤含有更多 种抗性基因，以多耐药性抗性基因和内酰胺类抗生素 抗性基因为主; 同时抗性基因的多样性和相对丰度受 铜浓度的影响显著，在中度污染 $(100 \sim 200 \mathrm{mg} / \mathrm{kg})$ 的红 土和重度污染 $(400 \sim 800 \mathrm{mg} / \mathrm{kg})$ 的潮土中最高，且与可 移动基因元件的丰度呈显著正相关; 结构方程模型进 一步证明，铜污染通过影响土壤中的微生物群落和可 移动基因元件驱动了抗性基因的变化，表明田间实际 条件下，长期铜污染将会极大地改变土壤中抗性基因 的多样性、丰度并促进传播. 在污染物的胁迫下，土 壤中的抗性菌会通过选择过程进行富集，并成为土壤 微生物中的优势菌群. 这些抗性菌中的ARGs又可以 通过水平基因转移(horizontal gene transfer)传递到敏 感菌，在土壤微生物群中传播抗性. 因此，土壤是 ARGs内在抗性和获得抗性的一个重要储存库, 土壤
的安全对人类和星球的健康变得越来越重要，也逐步 受到重视.

由于土壤微生物组中内在抗性的普遍存在, 因此, 区分自然产生的抗生素抗性基因与人为污染的抗生素 抗性基因(包括类型、含量、传播、风险、效应等)显 得尤其重要. 许多抗生素抗性基因与临床以及农业中 抗生素的使用密切相关，自然与人为污染产生的抗性 基因可以得到一定程度的区分，但对其生态风险还未 有系统的研究.

\section{3 土壤动物微生物组中的抗性组：一个“隐藏” 的库}

土壤中生活着丰富的、种类多样的动物，它们是 土壤生态系统的重要组成部分. 在长期的演化过程中, 这些动物体内往往定殖着独特的微生物类群. 近些年, 土壤动物微生物组研究受到中国学者的广泛关注, 初 步取得了一些阶段性成果. 土壤动物体内独特的生态 位往往使得其微生物类群不同于周围环境 ${ }^{[32]}$. 研究发 现, 不同于其他无脊椎动物(如蜜蜂 ${ }^{[33]}$ 、果蝇 ${ }^{[34]}$ 等), 土 壤动物体内含有丰富的微生物类群 ${ }^{[35]}$, 且在土壤动物 如跳虫肠道中已分离到丰富多样的抗菌物质 ${ }^{[36]}$. 这提 示, 土壤动物微生物组中可能含有多样的抗性组(图2). 一项关于野外不同土地利用方式的调查研究显示, 典 型土壤动物跳虫微生物组中含有丰富多样的抗性基 因，且土地利用方式可以影响其体内抗性基因的组 成 ${ }^{[35]}$. 进一步分析表明，其体内的抗性基因丰度与多 样性与土壤的施肥历史紧密相关. 在一项位于山东德 州的长期污泥农用的野外定位实验研究中, 学者们也 发现，长期施用污泥或鸡粪显著增加了土壤动物蚯蚓 肠道中的抗性基因丰度 ${ }^{[37]}$. 同时室内培养实验也证实, 猪粪施用后会显著增加土壤动物线虫、跳虫和捕食性 螨微生物组中的抗性组, 且抗性基因会在土壤食物链

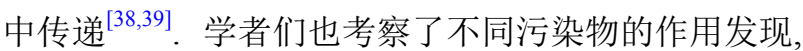
抗生素会显著增加土壤动物肠道中的抗性基因丰度, 但纳米银可减少土壤动物肠道中的抗性基因丰 度 ${ }^{[40,41]}$. 这表明, 不同污染物对土壤动物肠道微生物的 影响不同，但需要更多的研究揭示其抗性组改变的机 制. 此外，土壤食物网中抗性基因丰度的增加会产生 何种生态风险需要进一步的研究. 土壤动物微生物组 中的抗性组也可能通过其活动扩散到其他区域如根 际, 从而影响植物微生物组中的抗性组. 总体来说, 目 


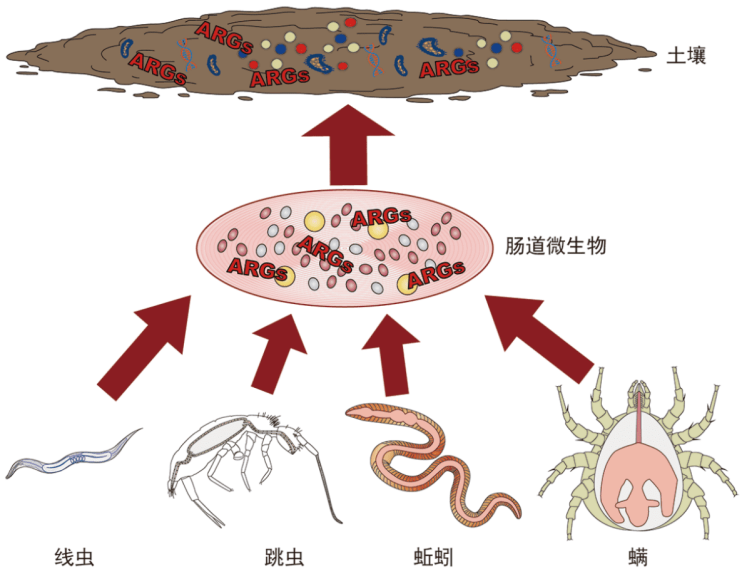

图 2 土壤动物微生物组中的抗性组

Figure 2 Antibiotic resistome in the soil animal microbiome

前已经初步揭示了主要土壤动物微生物组中的抗性 组, 但对于抗性组变化的深层次生态风险还未有系统 深入的探讨. 由于土壤动物微生物组也是土壤微生物 组的重要组成部分, 其抗性基因的变化将影响全球的 抗性基因的动态, 从而影响星球健康.

\section{4 土壤病毒组中的抗性组: 一个“移动”的库}

土壤是微生物的主要栖息地，微生物受到各种各 样的病毒侵染，因此土壤也是病毒的主要分布场所. 且病毒是地球上最丰富的生物实体，土壤病毒组是土 壤微生物组的重要组成部分 ${ }^{[42]}$. 其中噬菌体是土壤中 最丰富的病毒类群. 多项研究已经表明, 环境样品的 噬菌体基因组携带有丰富多样的抗性基因，可作为 ARGs的一个重要储存库 ${ }^{[43,44]}$. 例如, 从新鲜收割的蔬 菜(生菜、菠菜和黄瓜)和农田土壤分离得到的噬菌体 中均能检测到抗性基因 ${ }^{[43]}$; 在对使用牛粪和污泥进行 改良土壤的研究中，也在其噬菌体中检测到抗性基 因 ${ }^{[45]}$. 同时在选择压力下, 噬菌体可以介导ARGs的水 平转移, 又是 $A R G s$ 的一个可移动的库 ${ }^{[44]}$. 我国学者研 究发现, 噬菌体中tet基因的丰度与细菌中tet的丰度显 著正相关 ${ }^{[46]}$. 同时考虑到噬菌体在土壤中具有相当高 的丰度，其促进ARGs在土壤细菌中转移的作用不容 忽视，未来的研究应该对其有更多的关注. 土壤抗性 基因的研究到现阶段，已基本明确土壤中抗性基因的 种类与丰度，但对其可移动部分抗性基因的认知还很 缺乏，因此很难对抗性基因的风险进行合理评估，而
土壤病毒组中抗性基因的研究可以为其提供合适的基 础数据与理论支持.

\section{2 植物微生物组中的抗生素抗性组}

\section{1 植物微生物组}

植物和所有的真核生物一样被周围环境中的微生 物包围. 这些微生物包括细菌、真菌和原生生物, 可以 定殖于植物的表面和组织内部, 对宿主植物的功能和 环境适应性发挥着至关重要的作用. 这些微生物以及 所包含的遗传信息统称为植物微生物组 (plant micro-

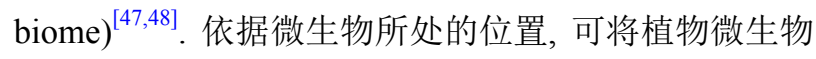
组分为叶际微生物组(phyllosphere microbiome)、根际 微生物组(rhizosphere microbiome) 以及内生微生物组 (endophytes microbiome). 叶际微生物是指定殖于植物 的地上部分或暴露于空气中的植物组织或器官包括茎 表面(caulosphere)、花表面(anthosphere)、果实表面 (carposphere)和叶表面(phylloplane) ${ }^{[49 \sim 51]}$ 的微生物. 全 球植物的叶际面积估算约 $10^{9} \mathrm{~km}^{2}$, 几乎是陆地面积的 两倍, 其上定殖有数百种微生物, 其中大部分为细菌, 而且仅细菌本身就达 $10^{6} \sim 10^{7} \mathrm{cells} / \mathrm{cm}^{2[49]}$, 而在全球范 围内叶际细菌的丰度可能超过 $10^{26} \mathrm{cells}^{[52]}$. 目前尚缺 乏关于叶际微生物中真菌丰度的数据, 但估计要比细 菌低得多. 不同植物叶际微生物群落组成差异性很大, 并易受到周围环境包括水分、气流、阳光辐射等因子 的影响. 此外, 叶际微生物被认为是植物与大气环境交 互响应的媒介 ${ }^{[53]}$, 也与人类微生物组间发生密切的交 流. 根际微生物定殖于植物根系周围的土壤, 其周围 土壤受到根系分泌物的影响 ${ }^{[54]}$. 根际微生物的数量远 远超过宿主植物本身, 根际微生物的数量可达 $10^{11}$ cells $/ \mathrm{g}$ 根, 原核微生物的种类超过 30000 种 ${ }^{[55]}$. 根际微 生物因土壤类型或植物类型的不同而不同, 根际微生 物群落的形成由土壤理化性质、植物根系分泌物、植 物内生菌和植物激素等因素共同影响 ${ }^{[56,57]}$. 植物内生 微生物定殖于植物组织内部, 包括细菌和真菌. 植物 内生微生物的多样性主要受寄主植物种类的影响, 此 外，外部环境包括土壤性质也可能对内生微生物产生 影响.

\section{2 植物微生物组与宿主的关系}

植物微生物组被认为是植物的“第二基因组”(sec- 
ondary genome)对植物的表型包括植物生长和病虫、 病原微生物的抵抗起着重要的作用. 由于植物微生物 组与植物本身密不可分, 近年来共生功能体(holobiont)的概念被提出, 以表明植物微生物与宿主植物的

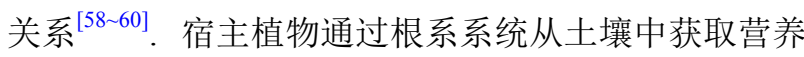
元素, 在功能上类似于动物的肠道. 越来越多的研究 表明, 根际微生物的组成和遗传信息的多样性以及与 宿主植物的相互作用, 对植物的生长和降低对病虫害 (包括病毒、病原菌、真菌及线虫)的敏感性起重要作 用 ${ }^{[61,62]}$. 植物微生物可以增加宿主微生物的表型从而 增强宿主对环境的适应能力. 研究表明, 宿主植物可 能进化出一些招募以及维持植物微生物组的能力, 比 如诱导、刺激或抑制等行为. 因此, 植物和植物微生 物组直接存在着共进化的关系 ${ }^{[63]}$.

\section{3 植物微生物组与人类健康}

植物微生物组是连接人类肠道微生物组和环境微 生物组的桥梁, 也是人类接触环境抗生素抗性组(resistome)的主要途径之一 ${ }^{[64]}$. 目前, 关于抗生素抗性组在 非临床环境中的研究主要集中于土壤和水体环 境 ${ }^{[24,65]}$, 关于植物微生物组对抗性基因扩散的影响仍 处于起步阶段. 植物微生物对抗性基因的传播可通过 食物链传递和绿色空间接触进行. 食物摄入是人类接 触环境微生物组和抗性组的主要方式, 这一过程中植 物微生物组起着重要的作用. 研究表明, 植物可以吸 收施用过有机肥土壤中的抗生素, 从而对植物微生物 组中的抗性基因起到选择和富集的作用. 此外，一些 含有抗性基因的微生物可以通过土壤-根系系统最终 进入到植物的内生微生物组和叶际微生物组. 我国学 者通过高通量苂光定量PCR 在植物微生物组中检测到 上百个抗性基因, 并且发现有机肥的施用显著增加了 叶际微生物中抗性基因的多样性和丰度 ${ }^{[66]}$. 一项最近 的研究也表明, 粪肥的施用可以增加植物微生物组中 的抗性基因, 且存在人类食物链传递的风险 ${ }^{[67]}$. 大部 分含有抗性基因的植物微生物不具有致病性(nonpathogenic), 但通过水平转移这些抗性基因可能扩散 到人类的致病菌中, 从而对人类的健康构成潜在的威 胁. 随着城市化水平的不断提高, 城市绿色空间(公园) 成为人类活动和娱乐的重要场所之一. 研究表明, 人类 多接触自然环境可以改变人类微生物组的多样性有益 于人体健康. 但也有研究表明, 城市绿色空间的植物微
生物组中含有大量的抗性基因，比如在城市绿色空间 中的草坪微生物组中检测到超过200种抗性基因，而 这些抗性基因的分布与人类活动尤其是工业的分布显 著相关 ${ }^{[68]}$. 此外, 大量的抗性基因在城市中水灌溉的 公园土壤中被检测到 ${ }^{[69]}$, 这些抗性基因也可能通过水 平转移进入到植物微生物组中, 从而增加了人类与抗 性基因和抗性微生物的接触. 因此, 城市绿色空间中 的微生物和抗性基因应该引起足够的重视和关注, 这 也与星球健康密切相关.

\section{3 抗性基因在土壤-微生物-动物-植物四元 体系中的流动}

如上所述, 植物微生物组是环境抗性基因的一个 重要储存库, 而土壤微生物组是植物微生物组的重要 来源. 植物微生物可通过与土壤微生物的“交流”获得 抗性基因, 从而使得抗性基因在土壤-植物系统中流 动, 并且通过食物链传递最终进入人类微生物组, 对 人类的健康造成潜在危害, 影响星球健康(图3).

\section{1 土壤微生物组和植物微生物组的交流}

土壤微生物组具有地球上最为复杂的群落, 它们 为植物提供了丰富的“微生物种子库”, ${ }^{60]}$. 土壤微生物 向植物的迁移主要通过根际到达根表和叶际, 最终进 入植物体内 ${ }^{[47]}$. 根际被看作是植物微生物组的“生长 室”. 这1 2 mm 的狭小空间是植物-微生物交互作用最 为强烈的区域. 植物根系通过根际沉积将大量的营养 物质和能量输入到土壤, 增强了土壤微生物活性并形 成了特异性的微生物群落, 同时微生物活性的增强加 快了营养元素的循环从而促进植物对营养元素的吸 收 ${ }^{[70]}$. 根际也因此成为土壤中微生物活动的热区, 并 促进了微生物向根表移动 ${ }^{[56]}$. 根表是土壤微生物特殊 的栖息地或者微生物移动的过渡边界，仅有一些土壤 微生物在经过根际选择和根表特异环境的二次选择后 最终栖息于此 ${ }^{[71]}$. 另外, 植物根系在其生长过程中会 导致根表细胞的分离, 从而为根际微生物或者根表微 生物进入植物体内提供了通道，一部分土壤微生物在 经过重重的选择后成为植物的内生菌 ${ }^{[71]}$. 叶际也是土 壤微生物和植物微生物交流的重要场所, 叶际微生物 往往要暴露于剧烈波动的温度、湿度、光照条件和贫 营养环境下，其多样性往往要低于土壤微生物和根际 


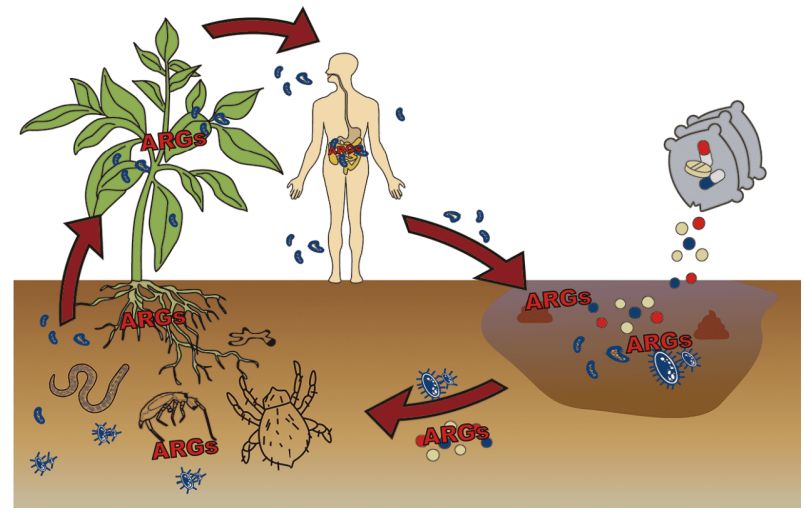

图 3 土壤生态系统中抗性基因的扩散

Figure 3 Dispersal of antibiotic resistance genes in the soil ecosystem

微生物 ${ }^{[72]}$. 除去大气来源，土壤微生物仍然是其组成 的重要部分 ${ }^{[49]}$. 灌溉以及土壤扬尘都会造成一部分土 壤微生物栖息于植物叶际. 同时，土壤微生物也会沿 着植物根、茎迁移至叶际 ${ }^{[73]}$. 土壤动物在土壤微生物 组和植物微生物的交流中也扮演着重要角色. 土壤动 物在其迁移或者取食过程中会增加土壤微生物向植物 的迁移. 例如, 研究表明, 线虫对根系的取食会为土壤 微生物进入植物体开辟通道 ${ }^{[74]}$.

\section{2 土壤抗性组和植物抗性组的相互作用}

土壤微生物组与植物微生物组的交流会直接导致 含有抗生素抗性基因的抗性菌从土壤微生物组向植物 微生物组的迁移, 特别是含有高丰度抗性基因的有机 肥施入土壤后，会促进抗性菌向植物微生物组的迁 移 ${ }^{[64]}$. 陈青林等人 ${ }^{[75]}$ 通过长期定位实验发现，污泥和 鸡粪的长期施用增加了玉米叶际抗生素抗性基因的多 样性和丰度. 最近的盆栽实验进一步证实, 猪粪的添加 增加了小麦和水稻叶表的抗性基因的多样性和丰 度 ${ }^{[76]}$. 进一步分析发现，这些抗性基因的增加可能来 源于有机肥中的抗性基因. 比如，陈青林等人 ${ }^{[66]}$ 利用 鸟粪石添加实验发现，叶际中30类抗生素抗性基因与 鸟粪石中的抗生素抗性基因专一性地共享, 说明鸟粪 石中抗性基因通过土壤微生物组和植物微生物组的交 流进入植物微生物组. 同样有研究发现, 有机肥施用后 抗生素抗性基因sulI, sulII, tet $\mathrm{C}$ 和 $\operatorname{tet} \mathrm{G}$ 在生菜的内生菌 及其叶际微生物中都有检测到，从而证实了抗性菌从 植物体向叶际的传输途径 ${ }^{[77]}$. 以上研究都证实，土壤 微生物和植物微生物以及植物的地下微生物和地上微
生物间的相互联系, 这些联系是抗性基因在植物-土壤 系统中流动的潜在途径.

基因的水平转移是环境中抗性基因扩散的重要方 式之一. 相关研究在多种植物的叶际都发现了不同种 类的可移动遗传元件, 并且其丰度与抗生素抗性基因 的丰度存在显著的正相关性, 说明基因的水平转移造 成了抗生素抗性基因在叶际的扩散 ${ }^{[5]}$. 已有研究证实, 植物叶际环境有利于质粒的水平转移, 从而加速抗生 素抗性基因在土壤-植物系统中的流动 ${ }^{[78]}$. 以上研究说 明, 基因的水平转移有助于抗生素抗性基因在土壤-植 物系统中的流动.

\section{3 抗性基因在土壤生态系统中流动的潜在健康 风险}

叶菜类蔬菜的生食是土壤抗性菌进入人体的潜在 途径 ${ }^{[79]}$. 研究表明, 有机生产的蔬菜中检测到的抗性 基因要远远大于普通蔬菜，其丰度可能是普通蔬菜的 8倍, 并且这些抗性基因涵盖了所有种类的抗生素抗 性基因 ${ }^{[80]}$. 另外, 这些用于上述实验的生菜直接购买 于普通的超级市场，进一步说明这种潜在风险的广泛 存在. 人类对生食蔬菜的需求是不可避免的, 清洗可 能是减少这种风险的有效途径, 而从源头上治理即减 少抗性基因在土壤-植物系统中的流动才是控制和减 少人体暴露抗性基因的根本方式 ${ }^{[64]}$. 因此, 未来研究 中需要更多地关注土壤-植物系统中抗性基因的流动.

\section{4 通过调控土壤-微生物-动物-植物系统中 的抗性基因流保证星球健康}

土壤(土壤-微生物-动物)-植物系统是自然环境与 人类健康联系的重要途径, 且通过食物链传递可直接 影响人类健康. 虽然土壤、动物和植物微生物组中天 然含有大量的抗菌物质(如抗生素)和抗性基因, 但是 人类活动(如滥用抗生素)正在加速抗性基因在土壤-微 生物-动物-植物系统中的富集与扩散, 并影响人类乃 至星球的健康 ${ }^{[64,81]}$. 为此, 本团队 ${ }^{[81]}$ 系统地阐述了土 壤生物如何通过抗性基因影响星球健康, 这是国际上 第一次明确地提出土壤生物与星球健康相联系的观 点. 同时, 对于如何调控土壤-微生物-动物-植物系统中 的抗性基因, 我国学者们也进行了一些尝试. 减少饲料 中抗生素的使用、将粪肥制成生物炭、调控微生物多 
样性以及噬菌体疗法可能是目前控制抗性基因污染较 为有效的手段.

通过比较使用抗生素与控制抗生素使用的猪粪农 用发现, 控制抗生素使用的处理中, 水稻和小麦叶际的 抗性基因丰度显著低于正常使用抗生素的处理 ${ }^{[76]}$. 这 表明控制饲料中抗生素的使用是控制抗性基因污染的 一个最为直接的手段. 周雪等人 ${ }^{[82]}$ 的研究表明, 如果 粪肥中含有较高丰度的抗性基因, 将猪粪等有机物料 烧制成生物炭，再进行农用可以显著降低有机肥施用 所带来的抗性基因污染风险. 陈青林等人 ${ }^{[83]}$ 也通过盆 栽实验表明, 生物炭的施用可以降低土壤和作物中抗 性基因的丰度. 安新丽等人 ${ }^{[84]}$ 的研究进一步表明, 生 物炭的添加可以减少一类整合子基因盒中的抗性基因 丰度，这将减少抗性基因转移的风险。最近丁晶等 ${ }^{[31]}$ 的研究表明, 虽然生物炭可以减少土壤中的抗性 基因丰度，但由于猪粪污泥等材料中往往含有丰富的 重金属等物质可能引起土壤动物肠道内抗性基因增加 的风险. 这些研究表明, 将猪粪等有机物料烧制成生物 炭, 虽然可以明确降低土壤和植物微生物组中的抗性 基因丰度, 但是可能增加取食生物炭的土壤动物肠道 中的抗性基因增加的风险. 调控土壤微生物及动物的 多样性也是管理抗性基因流的一项重要途径. 陈青林 等人 ${ }^{[85}$ 的研究表明, 土壤微生物多样性可明显影响抗 性基因的扩散. 朱冬等人 ${ }^{[86]}$ 的研究表明, 土壤动物跳 虫的活动会增加猪粪引入的抗性基因的扩散. 此外, 噬菌体疗法控制抗性基因最近得到了国内外学者的关 注, 但这些研究仍在起步阶段 ${ }^{[44]}$. 土壤生态系统中的 生物是相互联系的, 一个问题的解决需要考虑多种因 子的相互作用. 因此, 需要在土壤-微生物-动物-植物
整体系统中管理抗性基因流，从而更加全面地保证星 球健康.

\section{5 展望}

抗性基因污染是一个全球性的问题, 但目前我国 的研究还比较分散且多集中于调查类研究, 未来还需 要更多机理性的思考与探讨. 特别是以下 5 个方面, 未 来需要特别关注.

（1）虽然已经证实土壤-植物系统是抗性基因传播 的潜在重要途径, 但是对土壤-植物系统中抗性基因传 播的关键问题尚不清楚，如发生高频传播的抗性基因 类型、不同土壤-植物系统中抗性基因传播的共性和 个性, 以及土壤动物对抗性基因传播的影响等.

(2) 现有研究主要关注抗性基因的整体丰度, 对其 可移动部分抗性基因的丰度与风险仍知之甚少, 且对 抗性基因转移进入典型或潜在病原菌的频率也不 清楚.

(3) 农用抗生素也是环境抗生素的重要组成部分, 且大多为生物农药, 来源于微生物, 在使用过程中直接 作用于植物, 进入土壤. 虽有研究表明其可促进抗性基 因的产生, 但未有系统深入的研究, 未来应得到更多的 关注.

（4）抗性基因在环境中传播的风险已被认识, 但如 何客观地评价其健康风险并制定相应标准是面临的一 个挑战.

(5) 如何控制抗性基因在环境中的传播, 中国科学 家们进行了多种尝试, 但这些措施的野外效果如何, 以 及土壤性质对其的影响仍需要更多的研究与实践.

\section{参考文献}

1 Pruden A, Pei R, Storteboom H, et al. Antibiotic resistance genes as emerging contaminants: Studies in northern Colorado. Environ Sci Technol, 2006, 40: 7445-7450

2 Poirel L, Hombrouck-Alet C, Freneaux C, et al. Global spread of New Delhi metallo- $\beta$-lactamase 1. Lancet Infect Dis, 2010, 10: 832

3 Zhang N, Li M, Liu X. Distribution and transformation of antibiotic resistance genes in soil (in Chinese). China Environ Sci, 2018, 38: 26092617 [张宁, 李氷, 刘翔. 土壤中抗生素抗性基因的分布及迁移转化. 中国环境科学, 2018, 38: 2609-2617]

4 Müller D B, Vogel C, Bai Y, et al. The plant microbiota: Systems-level insights and perspectives. Annu Rev Genet, 2016, 50: 211-234

5 Blau K, Bettermann A, Jechalke S, et al. The transferable resistome of produce. mBio, 2018, 9: e01300-18

6 Zhu Y G, Gillings M, Simonet P, et al. Microbial mass movements. Science, 2017, 357: 1099-1100

7 Al-Delaimy W K. Planetary health and population health: The anthropocene requires different thinking and approaches in serving public health. Curr Envir Health Rpt, 2018, 5: 397-400 
8 Cole J, Bickersteth S. What's planetary about health? An analysis of topics covered in The Lancet Planetary Health's first year. Lancet Planet Health, 2018, 2: e283-e284

9 Nakandalage N, Nicolas M, Norton R M, et al. Improving rice zinc biofortification success rates through genetic and crop management approaches in a changing environment. Front Plant Sci, 2016, 7: 764

10 Seltenrich N. Down to earth: The emerging field of planetary health. Environ Health Perspect, 2018, 126: 072001

11 Li J W H, Vederas J C. Drug discovery and natural products: End of an era or an endless frontier? Science, 2009, 325: 161-165

12 Culp E J, Yim G, Waglechner N, et al. Hidden antibiotics in actinomycetes can be identified by inactivation of gene clusters for common antibiotics. Nat Biotechnol, 2019, 37: 1149-1154

13 Hautbergue T, Jamin E L, Debrauwer L, et al. From genomics to metabolomics, moving toward an integrated strategy for the discovery of fungal secondary metabolites. Nat Prod Rep, 2018, 35: 147-173

14 Sanderson H, Fricker C, Brown R S, et al. Antibiotic resistance genes as an emerging environmental contaminant. Environ Rev, 2016, 24: 205218

15 Allen H K, Donato J, Wang H H, et al. Call of the wild: Antibiotic resistance genes in natural environments. Nat Rev Microbiol, 2010, 8: 251-259

16 Allen H K, Moe L A, Rodbumrer J, et al. Functional metagenomics reveals diverse $\beta$-lactamases in a remote Alaskan soil. ISME J, 2009, 3: 243251

17 Galán J C, González-Candelas F, Rolain J M, et al. Antibiotics as selectors and accelerators of diversity in the mechanisms of resistance: From the resistome to genetic plasticity in the $\beta$-lactamases world. Front Microbio, 2013, 4: 9

18 Peterson E, Kaur P. Antibiotic resistance mechanisms in bacteria: Relationships between resistance determinants of antibiotic producers, environmental bacteria, and clinical pathogens. Front Microbiol, 2018, 9: 2928

19 An X L, Su J Q, Li B, et al. Tracking antibiotic resistome during wastewater treatment using high throughput quantitative PCR. Environ Int, 2018, 117: $146-153$

20 Wang F H, Qiao M, Lv Z E, et al. Impact of reclaimed water irrigation on antibiotic resistance in public parks, Beijing, China. Environ Pollut, 2014, 184: 247-253

21 Wolters B, Fornefeld E, Jechalke S, et al. Soil amendment with sewage sludge affects soil prokaryotic community composition, mobilome and resistome. FEMS Microbiol Ecol, 2019, 95

22 Zhao Y, Su J Q, An X L, et al. Feed additives shift gut microbiota and enrich antibiotic resistance in swine gut. Sci Total Environ, 2018, 621: $1224-1232$

23 Knapp C W, Dolfing J, Ehlert P A I, et al. Evidence of increasing antibiotic resistance gene abundances in archived soils since 1940. Environ Sci Technol, 2010, 44: 580-587

24 Zhu Y G, Johnson T A, Su J Q, et al. Diverse and abundant antibiotic resistance genes in Chinese swine farms. Proc Natl Acad Sci USA, 2013, 110: $3435-3440$

25 Li J, Cao J, Zhu Y G, et al. Global survey of antibiotic resistance genes in air. Environ Sci Technol, 2018, 52: 10975-10984

26 Zhou H, Wang X, Li Z, et al. Occurrence and distribution of urban dust-associated bacterial antibiotic resistance in Northern China. Environ Sci Technol Lett, 2018, 5: 50-55

27 Zeng Q T. Study on the contamination of typical antibiotic resistance genes (ARGs) in agricultural soil and the soil-air migration (in Chinese). Dissertation for Master's Degree. Hangzhou: Zhejiang University, 2019 [曾庆涛. 农田土壤典型抗生素抗性基因污染及其土-气迁移研究. 硕 士学位论文. 杭州: 浙江大学, 2019]

28 Zhang J, Sui Q, Tong J, et al. Soil types influence the fate of antibiotic-resistant bacteria and antibiotic resistance genes following the land application of sludge composts. Environ Int, 2018, 118: 34-43

$29 \mathrm{Hu} \mathrm{H} \mathrm{W}$, Wang J T, Li J, et al. Field-based evidence for copper contamination induced changes of antibiotic resistance in agricultural soils. Environ Microbiol, 2016, 18: 3896-3909

$30 \mathrm{Hu} \mathrm{H} \mathrm{W}$, Wang J T, Li J, et al. Long-term Nickel contamination increases the occurrence of antibiotic resistance genes in agricultural soils. Environ Sci Technol, 2017, 51: 790-800

31 Ding J, Yin Y, Sun A Q, et al. Effects of biochar amendments on antibiotic resistome of the soil and collembolan gut. J Hazard Mater, 2019, 377: $186-194$

32 Berg M, Stenuit B, Ho J, et al. Assembly of the Caenorhabditis elegans gut microbiota from diverse soil microbial environments. ISME J, 2016, 
10: 1998-2009

33 Engel P, Martinson V G, Moran N A. Functional diversity within the simple gut microbiota of the honey bee. Proc Natl Acad Sci USA, 2012, 109: 11002-11007

34 Adair K L, Wilson M, Bost A, et al. Microbial community assembly in wild populations of the fruit fly Drosophila melanogaster. ISME J, 2018, 12: 959-972

35 Zhu D, Chen Q L, Li H, et al. Land use influences antibiotic resistance in the microbiome of soil collembolans Orchesellides sinensis. Environ Sci Technol, 2018, 52: 14088-14098

36 Agamennone V, Roelofs D, van Straalen N M, et al. Antimicrobial activity in culturable gut microbial communities of springtails. J Appl Microbiol, 2018, 125: 740-752

37 Ding J, Zhu D, Hong B, et al. Long-term application of organic fertilization causes the accumulation of antibiotic resistome in earthworm gut microbiota. Environ Int, 2019, 124: 145-152

38 Zhu D, Xiang Q, Yang X R, et al. Trophic transfer of antibiotic resistance genes in a soil detritus food chain. Environ Sci Technol, 2019, 53: $7770-7781$

39 Zheng F, Zhu D, Giles M, et al. Mineral and organic fertilization alters the microbiome of a soil nematode Dorylaimus stagnalis and its resistome. Sci Total Environ, 2019, 680: 70-78

40 Zhu D, An X L, Chen Q L, et al. Antibiotics disturb the microbiome and increase the incidence of resistance genes in the gut of a common soil collembolan. Environ Sci Technol, 2018, 52: 3081-3090

41 Zhu D, Zheng F, Chen Q L, et al. Exposure of a soil collembolan to Ag nanoparticles and $\mathrm{AgNO}_{3}$ disturbs its associated microbiota and lowers the incidence of antibiotic resistance genes in the gut. Environ Sci Technol, 2018, 52: 12748-12756

42 Pratama A A, van Elsas J D. The 'neglected' soil virome-Potential role and impact. Trends Microbiol, 2018, 26: 649-662

43 Larrañaga O, Brown-Jaque M, Quirós P, et al. Phage particles harboring antibiotic resistance genes in fresh-cut vegetables and agricultural soil. Environ Int, 2018, 115: 133-141

44 Ye M, Sun M, Huang D, et al. A review of bacteriophage therapy for pathogenic bacteria inactivation in the soil environment. Environ Int, 2019, 129: 488-496

45 Ross J, Topp E, Schaffner D W. Abundance of antibiotic resistance genes in bacteriophage following soil fertilization with dairy manure or municipal biosolids, and evidence for potential transduction. Appl Environ Microbiol, 2015, 81: 7905-7913

Sun M, Ye M, Jiao W, et al. Changes in tetracycline partitioning and bacteria/phage-comediated ARGs in microplastic-contaminated greenhouse soil facilitated by sophorolipid. J Hazard Mater, 2018, 345: 131-139

47 Cordovez V, Dini-Andreote F, Carrión V J, et al. Ecology and evolution of plant microbiomes. Annu Rev Microbiol, 2019, 73: 69-88

48 Dini-Andreote F, Raaijmakers J M. Embracing community ecology in plant microbiome research. Trends Plant Sci, 2018, 23: 467-469

49 Vorholt J A. Microbial life in the phyllosphere. Nat Rev Microbiol, 2012, 10: 828-840

50 Meyer K M, Leveau J H J. Microbiology of the phyllosphere: A playground for testing ecological concepts. Oecologia, 2012, 168: 621-629

51 Zarraonaindia I, Owens S M, Weisenhorn P, et al. The soil microbiome influences grapevine-associated microbiota. mBio, 2015, 6: e02527-14

52 Lindow S E, Brandl M T. Microbiology of the phyllosphere. Appl Environ Microbiol, 2003, 69: 1875-1883

53 Farré-Armengol G, Filella I, Llusia J, et al. Bidirectional interaction between phyllospheric microbiotas and plant volatile emissions. Trends Plant Sci, 2016, 21: 854-860

54 Mendes R, Garbeva P, Raaijmakers J M. The rhizosphere microbiome: Significance of plant beneficial, plant pathogenic, and human pathogenic microorganisms. FEMS Microbiol Rev, 2013, 37: 634-663

55 Mendes R, Kruijt M, de Bruijn I, et al. Deciphering the rhizosphere microbiome for disease-suppressive bacteria. Science, 2011, 332: 1097-1100

56 Kent A D, Triplett E W. Microbial communities and their interactions in soil and rhizosphere ecosystems. Annu Rev Microbiol, 2002, 56: 211236

57 Sanon A, Andrianjaka Z N, Prin Y, et al. Rhizosphere microbiota interfers with plant-plant interactions. Plant Soil, 2009, 321: 259-278

Cregger M A, Veach A M, Yang Z K, et al. The Populus holobiont: Dissecting the effects of plant niches and genotype on the microbiome. Microbiome, 2018, 6: 31

59 Sánchez-Cañizares C, Jorrín B, Poole P S, et al. Understanding the holobiont: The interdependence of plants and their microbiome. Curr Opin Microbiol, 2017, 38: 188-196 
60 Vandenkoornhuyse P, Quaiser A, Duhamel M, et al. The importance of the microbiome of the plant holobiont. New Phytol, 2015, 206: 11961206

61 Yang J, Kloepper J W, Ryu C M. Rhizosphere bacteria help plants tolerate abiotic stress. Trends Plant Sci, 2009, 14: 1-4

62 Bakker P A H M, Doornbos R F, Zamioudis C, et al. Induced systemic resistance and the rhizosphere microbiome. Plant Pathol J, 2013, 29: 136143

63 Panke-Buisse K, Poole A C, Goodrich J K, et al. Selection on soil microbiomes reveals reproducible impacts on plant function. ISME J, 2015, 9: 980-989

64 Chen Q L, Cui H L, Su J Q, et al. Antibiotic resistomes in plant microbiomes. Trends Plant Sci, 2019, 24: 530-541

65 Baquero F, Martínez J L, Cantón R. Antibiotics and antibiotic resistance in water environments. Curr Opin Biotech, 2008, 19: 260-265

66 Chen Q L, An X L, Zhu Y G, et al. Application of struvite alters the antibiotic resistome in soil, rhizosphere, and phyllosphere. Environ Sci Technol, 2017, 51: 8149-8157

67 Zhang Y J, Hu H W, Chen Q L, et al. Transfer of antibiotic resistance from manure-amended soils to vegetable microbiomes. Environ Int, 2019, 130: 104912

68 Yan Z Z, Chen Q L, Zhang Y J, et al. Antibiotic resistance in urban green spaces mirrors the pattern of industrial distribution. Environ Int, 2019, 132: 105106

69 Wang F H, Qiao M, Su J Q, et al. High throughput profiling of antibiotic resistance genes in urban park soils with reclaimed water irrigation. Environ Sci Technol, 2014, 48: 9079-9085

70 Edwards J, Johnson C, Santos-Medellín C, et al. Structure, variation, and assembly of the root-associated microbiomes of rice. Proc Natl Acad Sci USA, 2015, 112: E911-E920

71 Bulgarelli D, Schlaeppi K, Spaepen S, et al. Structure and functions of the bacterial microbiota of plants. Annu Rev Plant Biol, 2013, 64: 807-838

72 Leveau J. Microbiology: Life on leaves. Nature, 2009, 461: 741-742

73 Bai Y, Müller D B, Srinivas G, et al. Functional overlap of the Arabidopsis leaf and root microbiota. Nature, 2015, 528: 364-369

74 Topalović O, Heuer H. Plant-nematode interactions assisted by microbes in the rhizosphere. Curr Issues Mol Biol, 2019, 30: 75-88

75 Chen Q L, An X L, Zheng B X, et al. Long-term organic fertilization increased antibiotic resistome in phyllosphere of maize. Sci Total Environ, 2018, 645: 1230-1237

76 Zhou S Y D, Zhu D, Giles M, et al. Phyllosphere of staple crops under pig manure fertilization, a reservoir of antibiotic resistance genes. Environ Pollut, 2019, 252: 227-235

77 Fang H, Wang H, Cai L, et al. Prevalence of antibiotic resistance genes and bacterial pathogens in long-term manured greenhouse soils as revealed by metagenomic survey. Environ Sci Technol, 2015, 49: 1095-1104

78 van Elsas J D, Turner S, Bailey M J. Horizontal gene transfer in the phytosphere. New Phytol, 2003, 157: 525-537

79 Sannes M R, Belongia E A, Kieke B, et al. Predictors of antimicrobial-resistant Escherichia coli in the feces of vegetarians and newly hospitalized adults in Minnesota and Wisconsin. J Infect Dis, 2008, 197: 430-434

80 Zhu B, Chen Q, Chen S, et al. Does organically produced lettuce harbor higher abundance of antibiotic resistance genes than conventionally produced? Environ Int, 2017, 98: 152-159

81 Zhu Y G, Zhao Y, Zhu D, et al. Soil biota, antimicrobial resistance and planetary health. Environ Int, 2019, 131: 105059

82 Zhou X, Qiao M, Su J Q, et al. Turning pig manure into biochar can effectively mitigate antibiotic resistance genes as organic fertilizer. Sci Total Environ, 2019, 649: 902-908

83 Chen Q L, Fan X T, Zhu D, et al. Effect of biochar amendment on the alleviation of antibiotic resistance in soil and phyllosphere of Brassica chinensis L. Soil Biol Biochem, 2018, 119: 74-82

84 An X L, Chen Q L, Zhu D, et al. Distinct effects of struvite and biochar amendment on the class 1 integron antibiotic resistance gene cassettes in phyllosphere and rhizosphere. Sci Total Environ, 2018, 631-632: 668-676

85 Chen Q L, An X L, Zheng B X, et al. Loss of soil microbial diversity exacerbates spread of antibiotic resistance. Soil Ecol Lett, 2019, 1: 3-13

86 Zhu D, Wang H T, Zheng F, et al. Collembolans accelerate the dispersal of antibiotic resistance genes in the soil ecosystem. Soil Ecol Lett, 2019, 1: $14-21$ 


\title{
Antibiotic resistance genes in the soil ecosystem and planetary health: Progress and prospect
}

\author{
ZHU Dong ${ }^{1}$, CHEN QingLin ${ }^{2}$, DING Jing ${ }^{1}$, WANG YiFei ${ }^{1}$, CUI HuiLing ${ }^{1}$ \& ZHU YongGuan ${ }^{1,2}$ \\ 1 State Key Laboratory of Urban and Regional Ecology, Research Center for Eco-Environmental Sciences, Chinese Academy of Sciences, \\ Beijing 100085, China; \\ 2 Key Lab of Urban Environment and Health, Institute of Urban Environment, Chinese Academy of Sciences, Xiamen 361021, China
}

The soil-plant system is an important route for antibiotic resistance genes (ARGs) to spread from the environment to human beings. Through the study of ARGs in the soil-plant system, their distribution and transmission in soil organisms can be clarified, and the risk and rule of the transmission of ARGs in the food chain can be revealed, which provide a theoretical basis for controlling the pollution of resistance genes and guarantee human health. Therefore, it is also of great value in maintaining planetary health. This paper firstly summarize the important research progress of resistome in the soil (including soil fauna gut) and plant microbiomes, then highlight the flow of resistance genes in the soil-plant system, and finally propose that planetary health can be ensured by managing the flow of resistance genes in the soil-plant system and discuss future research directions.

soil-plant system, planetary health, antibiotic resistance genes, food chain transfer, human activity

doi: $10.1360 /$ SSV-2019-0267 